\title{
Optimal Pole Number for Magnetic Noise Reduction in Variable Speed Permanent Magnet Synchronous Machines with Fractional-Slot Concentrated Windings
}

\author{
Pedram Asef ${ }^{1}$, Member, IEEE, Ramon Bargalló Perpiñà ${ }^{2}$, and Andrew C. Lapthorn², Member, IEEE, \\ ${ }^{1}$ Department of Electrical Engineering, Universitat Politècnica de Catalunya-BarcelonaTech, Barcelona 08019, Spain \\ ${ }^{2}$ Department of Electrical and Computer Engineering, University of Canterbury, Christchurch 8041, New Zealand
}

Increasing the number of pole-pairs leds to a lower electromagnetic yoke, and therefore lower vibration and magnetic noise occur. In this research, the influence of different numbers of pole-pairs on the vibro-acoustic design aspects of the machine is studied for the first time using a multi-slice subdomain method (MS-SDM) while considering the natural frequencies under a variable speed analysis. This study aims to determine the optimal number of pole-pairs for a lowspeed, high-torque permanent magnetic synchronous generator (PMSG) with double-layer, fractional-slot, nonoverlapping, concentrated windings (FSCW) for wind turbine applications. First, all possible slots per pole per phase combinations which offer the use of double-layer FSCW are studied through a magnetomotive force (MMF) harmonic analysis. Second, the MS-SDM of the PMSG is studied to examine the vibro-acoustic performance under a variable speed analysis. Finally, all affected major parameters are compared in order to find the optimal pole number of the PMSG. To verify the MS-SDM-based results, both 3-D finite element analysis and experimental investigations are employed.

Index Terms-Finite element analysis, magnetic noise, permanent magnet machines, resonance, subdomain model, vibration, variable speed.

\section{INTRODUCTION}

$I^{2}$ $\mathrm{N}$ fact, there are many empirical-based studies for calculating the optimum pole number for restricting the magnetic noise radiated from electrical machines. However, these studies have never considered the behavior of the machine under a variable speed analysis, regardless of the fact that resonances, and their effect on the choice of optimal pole number are occasionally critical. Several of these studies to reduce magnetic noise and vibrations using generic slot per pole combination can be addressed in [1], where the first attempts to study an acoustic design perspective were presented by Kron. In 1989, some of these laws are listed in Timar's book [2], such as $0.75 Z_{\mathrm{s}} \leq Z_{\mathrm{r}}<Z_{\mathrm{s}}$. These empirical laws, not only in the 19th century but also in the 20th, are globally proposed by researchers [3-4]. In addition, there are many recent publications [5-8] dealing with magnetic noise and vibration, where the designers have ignored the fact that electrical machines can run at variable speed. In addition to that, not considering natural frequencies can be critical when the operation point obtained by optimum parameters is nearby or inside the resonance regions.

During recent research developments in this field, the experts have found the importance of these considerations. Some of these studies can be addressed as follows.

In [9], the authors present an analytical subdomain model to compute the magnetic field distribution in surface-mounted permanent-magnet synchronous motors (PMSMs). The proposed model is sufficiently general to be applied with any number of slots per pole per phase (known as q) and any windings topology. The analytical method is based on the resolution of two-dimensional Laplace and Poisson equations in polar coordinates for each subdomain. In another work [10], the paper develops an analytical model for computing the radial vibration force of fractional-slot concentrated winding (FSCW) in PMSMs. In this work, the researchers present the impact of several important issues such as stator slotting, tangential field component, radius in the airgap for computation, and load condition on the vibration.

In research [11], the authors propose a fast simulation tool which is based on analytical models for the variable-speed magnetic noise emitted by induction machines. Finally, they proposed a power range tool based on a dataset, where the former empirical criterions were modified. Furthermore, the influence of vibration-based forces and noises due to a PWM supply in induction machines has been studied in [12-13], where they considered natural frequencies.

This paper investigates the impact of optimal pole number, in which a double-layer FSCW is employed. Therefore, there are not many $q$ combinations which are investigated to reduce the audible magnetic noise and vibration level on a low-speed, high-torque permanent magnet synchronous generator (PMSG) for a variable-speed-range analysis. The vibro-acoustic modeling of the PMSG is based on a multi-slice subdomain model (MS-SDM), in which the machine running at variable speed and the natural frequencies are considered. The results and improvements of each machine are studied in both the electromagnetic and vibro-acoustic design aspects. Finally, the optimum pole number is proposed to reduce magnetic noise and vibrations with respect to the electromagnetics consequences. The MS-SDM results are verified using both 3-D FEA and experimental tests with a very good agreement. This work is based on five MS-SDMs that were implemented in simulation tools, MANATEE linked to MATLAB, and ANSYS Maxwell as 3-D FEA package, is employed to validate the results.

\section{ANALYTICAL MMF HARMONIC ANALYSIS OF EACH MACHINE WITH DOUBLE-LAYER FSCWS}

The stator winding factor of PMSMs is studied for the 
fractional-slot concentrated windings (FSCW) with $q<1$ which depends on the slot/pole numbers. The fundamental winding factor $\left(k_{\mathrm{w} 1}\right)$ is reported in Table $\mathrm{I}$. The number of stator slots is fixed to 36 , because of weight and dimension restrictions. To obtain high performance non-overlapping FSCWs, 36 slots appears to be the best choice among other options. Regarding the outer and inner diameters of the generator, a larger number of slots than 36 could put the machine in a high risk of saturation, mainly in the teeth. Likewise, a smaller number of stator slots has weakened the electromagnetic performance of the PMSG with a low efficiency and power density. Moreover, other combination possibilities such as 24 slots resulted in zero mean EMF. The periodic or antiperiodic indicates the winding distribution. As the electromagnetic torque is a significant product of PMSMs and is proportional to the value of $k_{\mathrm{w} 1}$, therefore, a higher fundamental $k_{\mathrm{w} 1}$, when other sub-harmonics are as low as possible is preferred. To enhance a small $k_{\mathrm{w} 1}$ in special machines, a higher current or coil turns are required which makes the machine more expensive.

Table I illustrates that the study investigates five different pole-pairs which affect the stator winding distribution of each machine to offer benefits of the double-layer FSCWs. The winding factor of five different slot/pole combinations, where each of those machines (1-5) have shown various winding layouts and fundamental winding factors. Machine No. 4 with 36 slots, 40 poles has the highest $k_{\mathrm{wl}}=0.945214$, and apparently the best combination. The highest $k_{\mathrm{w} 1}$ brings the greatest electromotive forces (EMFs) and highest average electromagnetic torque. Machine No. 1 has the minimum $k_{\mathrm{w} 1}$, which meant the lowest production of EMFs and torque. All five machines have a periodic winding distribution, and also assumed to have equal winding turns and magnet volume for an identical comparison. These five machines are selected based on the defined optimization constraints for the design and experiment stage such as: a) satisfying $q$ for only nonoverlapping double-layer FSCW; b) electromagnetic capability, in which efficiency $g_{1}(x)=0.90-\eta \leq 0$, output power $g_{2}(\mathrm{x})=$ $1200-P_{\mathrm{o}} \leq 0$, current density $g_{3}(x)=J_{\mathrm{c}}-4 \leq 0$ (at rated speed of $150 \mathrm{rpm}$ ). From all possible pole numbers, only these five machines could successfully satisfy the design constraints.

Fig. 1 illustrates the winding layout of each three-phase machine, where the double-layer non-overlapping FSCW pattern is considered. Fig. 1(a) presents the winding pattern of machine No.1 with a winding layout of 'CA'AB'BA'AB'BC'CB'BC', Fig. 1(b) shows the winding pattern of machine No.2 with winding layout of ' $C A$ ' $A B$ ' $B C$ '. Fig. 1(c) indicates the machine No. 3 winding the stator winding pattern with the following layout 'AA'AB'BB'BC'CC'CA'. Fig. 1(d) represents the wind pattern of machine No. 4 with a layout of 'BA 'AA'AA'AC'CC'CC'CB'BB'BB'. Fig. 1(e) depicts the stator winding pattern of machine No. 5 with the layout of 'BA'AA'AC'CC'CB'BB'. Because of the discrete position of a coil in the slots, space harmonics are present in the MMF distribution, where $v$ is the order of the MMF harmonic based on the two-pole MMF harmonic, which known as the fundamental harmonic as well. Thus, the order of the main harmonics is $v=p$, where $p$ is the number of pole pairs [14-15]. The number $v$ is considered without a sign. It can be the only positive integer. The main harmonic is that harmonic whose order is the same as the number of $p$. Only this main harmonic is synchronous with respect to the rotor. Vice versa, the total harmonics of a various order are asynchronous with the rotor. Therefore, since they cause a time-varying magnetic field, they induce currents in the rotor, which is one of the most significant reasons that induced eddy-current losses (mostly in the rotor) can be increased along with a permeance variation and PWM harmonics [16]. Synthesis of different slot/pole combinations

TABLE I. DOUBLE-LAYER FSCW CHARACTERISTICS

\begin{tabular}{c|c|ccccc}
\hline \multicolumn{2}{c|}{$\begin{array}{c}\text { Slot/pole } \\
\text { number }\end{array}$} & $\begin{array}{c}\text { Slots/poles } \\
\text { combination }\end{array}$ & $q$ & $\begin{array}{c}\text { Periodic } \\
\text { number of } \\
\text { winding }\end{array}$ & $t$ & $k_{\mathrm{w} 1}(\mathrm{pu})$ \\
\hline 1 & $36 / 20$ & $9 / 5$ & $3 / 5$ & 12 & 2 & 0.735246 \\
2 & $36 / 24$ & $3 / 2$ & $1 / 2$ & 12 & 12 & 0.866025 \\
3 & $36 / 30$ & $6 / 5$ & $2 / 5$ & 6 & 3 & 0.933013 \\
4 & $36 / 40$ & $9 / 10$ & $3 / 10$ & 4 & 4 & 0.945214 \\
5 & $36 / 42$ & $6 / 7$ & $2 / 7$ & 6 & 3 & 0.933013 \\
\hline
\end{tabular}

Stator winding distribution of $\tau=1 / 1.8$ over 1 period (M. 1)

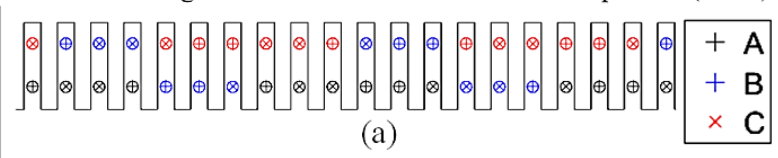

Stator winding distribution of $\tau=1 / 1.5$ over 1 period (M. 2)

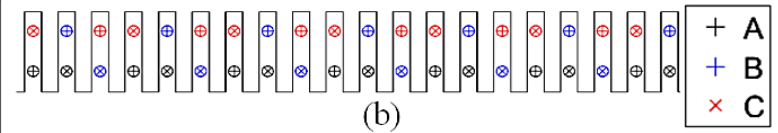

Stator winding distribution of $\tau=1 / 1.2$ over 1 period (M. 3)

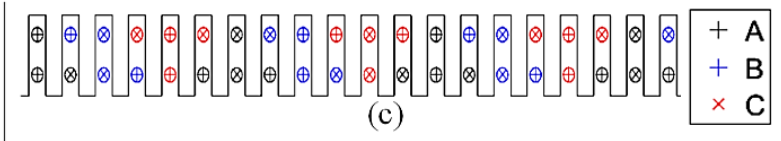

Stator winding distribution of $\tau=1 / 0.9$ over 1 period (M. 4)

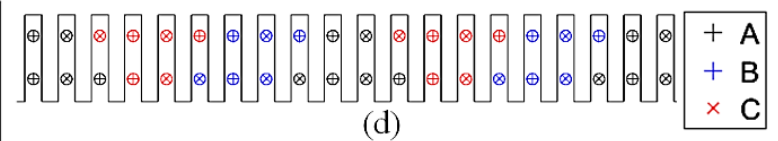

Stator winding distribution of $\tau=1 / 0.85714$ over 1 period (M. 5)

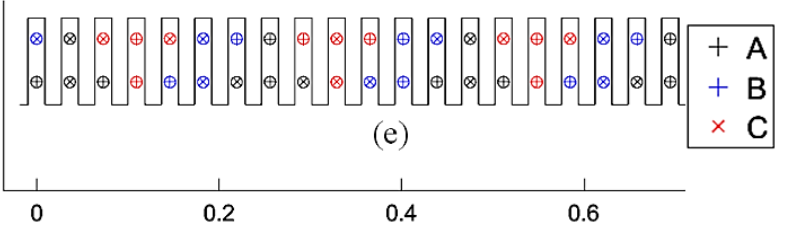

Position along air-gap in trigonometric direction [m]

Fig. 1. Double-layer non-overlapping FSCW distribution of each studied machine, (a) Machine No.1, (b) Machine No. 2, (c) Machine No. 3, (d) Machine No. 4, and (e) Machine No. 5.

with respect to the dimensions and an acceptable winding factor can be chosen in the design of FSCWs. As the PMSG is assumed to operate for wind power applications, and thus, nonoverlapping FSCW topology has become one of the prime design optimization constraint (which has considerably decreased the number of $q$ possibilities) due to its electromagnetic advantages over distributed winding topology such as: 1) higher power density; 2) higher efficiency; 3) lower cogging torque; 4) higher slot fill factor (appx. 60\%); this value was reachable because of the segmented stator; 5) shorter end turns, this comes with lower copper loss; 6) flux weakening 
capability which is very important in wind power applications due to a wide constant power range; 7) higher harmonic order for torque-producing, and 8) higher electric loading which affects the total power. Although, it should be mentioned that distributed windings offer a lower level of maximum noise and vibration because of lower number of wavenumbers $r$. However, this is a solvable issue for FSCWs by choosing smaller $q$ with larger number of poles which causes higher electromagnetic yoke height and $\operatorname{GCD}\left(Q_{\mathrm{s}}, 2 p\right)$. As a result, a more comprehensible noise and vibration levels can be seen. Next, to harmonics of higher order (fundamental) there are also lower order harmonics, which are known as sub-harmonics.

Fig. 2 illustrates the MMF behavior in the air-gap, which is enlarged for a better understanding in this graph. The fundamental or main harmonics $v_{3}=p$ are synchronous with the rotor speed, where the sub-harmonic $v_{1}<p$ occurs when the speed is greater than rotor speed. Whereas, the speed is lower than the rotor speed, positive or negative harmonics of $v_{2}>p$ can appear as shown in the figure. The MMF harmonics swivel in the air-gap by various speeds. The mechanical speed of the conventional $v$-th MMF harmonic in the stator reference plane is calculated from [16]:

$$
\omega_{v s}=\frac{\omega}{\operatorname{sign} \cdot v}
$$

where sign shows the sign function, and sign $=+1$ if the $v$-th harmonic rotates in the identical direction of the rotor, on the other hand, sign $=-1$ if the $v$-th harmonic rotates in the inverse direction. The sign function is defined in [16]; harmonics which correspond to the values as follow:

$$
v=1+3 k, \quad \text { where }: k=0,1,2, \ldots
$$

by the positive sign, when harmonics which correspond to the following quantities [16]:

$$
v=2+3 k, \quad \text { where }: k=0,1,2, \ldots
$$

have the negative sign, where $k$ is always a positive integer.

A positive sign is often assigned to the series including the main harmonic $(v=p)$. The MMF speed with respect to the rotor is computed as [16]:

$$
\omega_{r v}=2 \pi f\left(\frac{\operatorname{sign}}{v}-\frac{1}{p}\right)
$$

The rotating MMF harmonics induce in the rotor an EMF at the frequency $f_{\mathrm{rv}}$. This frequency is defined as a function of the harmonic order $v$ through [16]:

$$
f_{r v}=v \frac{\omega_{r v}}{2 \pi}=f\left|\operatorname{sign}-\frac{v}{p}\right|
$$

Fig. 2 presents the total air-gap MMF waveforms of each machine with the double-layer non-over lapping FSCWs as a function of space, in addition, the harmonic content over the wavenumber of each studied PMSG is individually shown. Machine No. 1 with total harmonic distortion (THD) of 0.492736 is presented in Fig. 2(a). Machine No. 2 with a larger THD of 0.556982 over each wavenumber is seen in Fig. 2(b). Machine No. 3 with the largest THD of 0.764972 is indicated in Fig. 2(c). Machine No. 4 as the second largest THD, 0.731797 is reported in Fig. 2(d). Machine No. 5 (shown in Fig. 2(e)) with THD of 0.700293 reinforces that there is no linear relationship between the pole-pairs number and the THD of the total air-gap MMFs.
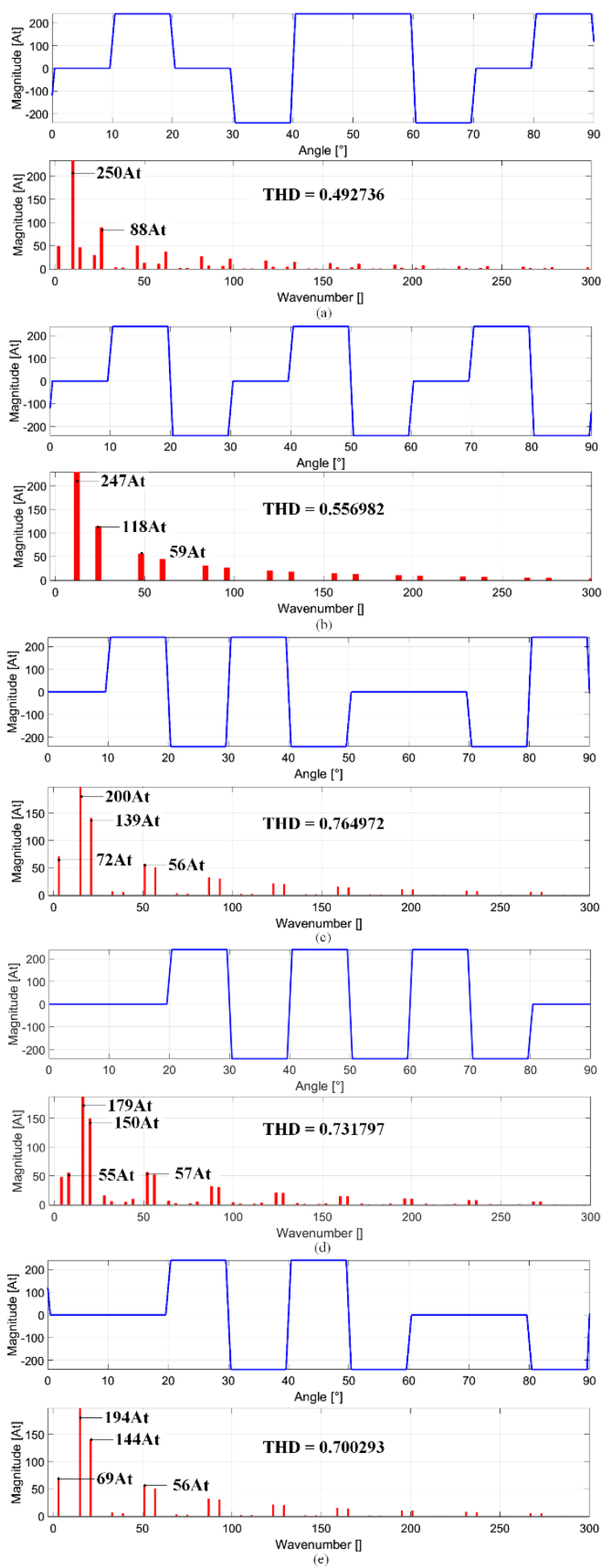

Fig. 2. Total air-gap phase MMF, and its harmonic content as function of space for different FSCWs pattern, (a) Machine No.1, (b) Machine No. 2, (c) Machine No. 3, (d) Machine No. 4, and (e) Machine No. 5.

\section{Multi-Slice Subdomain Method (MS-SDM) DEFINITION OF PMSG}

To compute the vibro-acoustic performance of all studied PMSGs, the MS-SDM of the PMSG with an outer rotor and surface mounted topology is discussed in this section. The conventional SDM cannot be used while the machine's parts have skew, therefore, the MS-SDM is required for higher accuracy. The MS-SDM relies on the analytical resolution of 
Maxwell equations, through several validated assumptions (details can be seen in [9-10] [18-19]). These assumptions are summarized as follows:

1. The problem is resolved into physical subdomains by Cartesian geometry, where uniform magnetic relative permeability and consisting a magnetic source in form of current density or magnet.

2. The problem can be solved by a 2-D Magnetic Vector Potential (MVP) formulation in each subdomain slice. The magnetic flux density $B$ derives from the MVP $A$ by $B=\operatorname{rot}$ $(A)$, and thus, it is unceasing known inside the subdomain (without mesh discretization). However, the MS-SDM considers 3-D effects such as skewing and end effect.

3. The problem is solved under a steady-state condition for each self-determining and sequential time-step. It should be mention that the rotor mechanical speed is assumed changeless.

4. Stator and rotor iron cores are limitlessly permeable. Regarding the continuity equations, the tangential field is null at each iron interface, which allows to assume the iron cores as external boundaries of the problem. The MVP and the flux density distributions are ignored in iron cores and magnetic saturation.

Fig. 3 depicts the MS-SDM of the studied outer rotor surface mounted PMSG, in which the following expressions are defined.

$$
\begin{aligned}
& \frac{\partial A_{\text {slot }}}{\partial r}=0 \\
& \left\{\begin{array}{c}
A_{\text {slot }}=A_{\text {gap }} \\
\frac{\partial A_{\text {slot }}}{\partial r}=\frac{\partial A_{g a p}}{\partial r}
\end{array}\right. \\
& \nabla^{2} \vec{A}_{\text {slot }}=\mu_{0} \vec{J}_{\text {slot }} \\
& \frac{\partial A_{\text {slot }}}{\partial \theta}=0 \\
& \frac{\partial A_{\text {gap }}}{\partial r}=0 \\
& \nabla^{2} \vec{A}_{m}=\mu_{0} \vec{\nabla} \Lambda \vec{M}_{j} \\
& \frac{\partial A_{m}}{\partial r}=0 \\
& \left\{\begin{array}{c}
A_{m}=A_{g a p} \\
\frac{\partial A_{m}}{\partial r}+\mu_{0} M_{\theta_{j}}=\frac{\partial A_{g a p}}{\partial r}
\end{array}\right.
\end{aligned}
$$

where $A_{\text {slot }}, A_{\text {gap }}$, and $A_{\mathrm{m}}$ are magnetic vectors potential on the $r$ and $\theta$ coordinates due to the presence of current in the slot, airgap, and magnet, respectively. Vector $J_{\text {slot }}$ denotes the current density in the slot. $\Delta$ is the offset in the tangential direction, $M$ is the magnetization vector of the $j$ th subdomain slice, $\mu_{0}$ is the permeability of vacuum. $\Lambda$ is the air-gap permeance per unit area [9-10] [18].

By defining the transient magnetic field for low frequency based on Maxwell's equation as follow:

$$
\left\{\begin{array}{c}
\nabla \times \frac{1}{\sigma} \nabla \times H_{j}+\frac{\partial B_{j}}{\partial t}=0 \\
\nabla . B_{j}=0
\end{array}\right.
$$

$\sigma$ is the conductivity of the material used, $H_{\mathrm{j}}$ and $B_{\mathrm{j}}$ are the magnetic field strength and magnetic field density at $j$ th subdomain slice of the machine. To improve the MS-SDM accuracy, the $H_{\mathrm{j}}$ and $B_{\mathrm{j}}$ are computed using a 2D FEA software.

The resulting magnetic noise originates from magnetostrictive and Maxwell forces which are essentially produced by the stator winding and rotor magnet's harmonic magnetic field which, can be defined as:

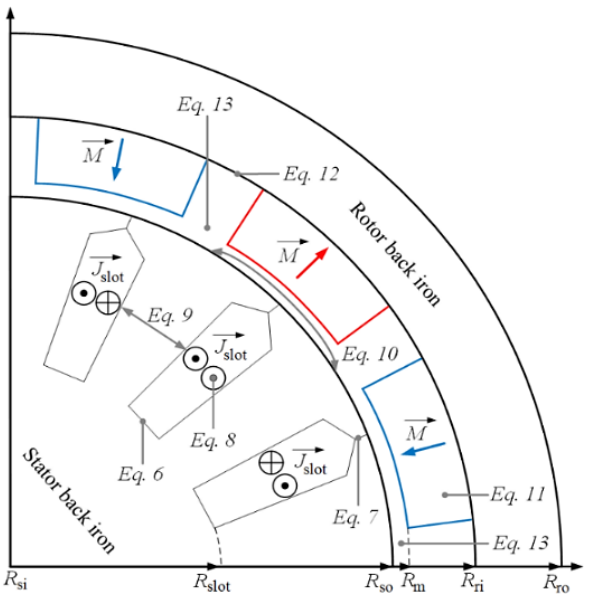

Fig. 3. The MS-SDM of the studied outer rotor PMSG.

$$
\left\{\begin{array}{c}
p_{r j}(\theta, z)=\frac{1}{2 \mu_{0}}\left(B_{r j}^{2}(\theta, z)-B_{\theta j}^{2}(\theta, z)-B_{z j}^{2}(\theta, z)\right) \\
p_{\theta j}(\theta, z)=\frac{B_{\theta j}(\theta, z) \cdot B_{r j}(\theta, z)}{\mu_{0}}
\end{array}\right.
$$

Hence, the sound wave of a PMSG as a useful idealistic homogeneous medium can be given at each $j$ th subdomain slice:

$$
\frac{\partial^{2} p_{j}}{\partial x^{2}}+\frac{\partial^{2} p_{j}}{\partial y^{2}}+\frac{\partial^{2} p_{j}}{\partial z^{2}}=\frac{1}{c^{2}} \frac{\partial^{2} p_{j}}{\partial t^{2}}
$$

The maximum sound power radiated by the PMSG due to magnetic vibrations can be also written as:

$$
W_{p j}\left(f_{\max }\right)=\frac{1}{2} Z_{0 j} S_{c j} \sigma_{m j}(f)\left\langle v_{m \omega}^{2}\right\rangle v_{m \omega}
$$

where $Z_{0 \mathrm{j}}$ denotes the air acoustic impedance, $S_{\mathrm{cj}}$ is the PMSG frame area, and $\sigma_{\mathrm{mj}}$ is the modal radiation factor at the $j$ th subdomain slice. The maximum sound power level $W_{\omega \mathrm{j}}$ can be computed using:

$$
W_{\omega j}\left(f_{\max }\right)=10 \log _{10}\left(\sum_{m} \frac{W_{p j}\left(f_{\max }\right)}{10^{-12}}\right)
$$

The natural frequencies of the stator circumferential mode have been only chosen as an even value due to the even stator and rotor pole numbers, and thus, the Maxwell exciting force harmonics have been produced as only even spatial orders due to symmetry reasons by assimilating the stator to an equivalent ring. The breathing mode natural frequency $f_{0}$ can be defined:

$$
f_{0}=\frac{1}{2 \pi a} \sqrt{\frac{E_{s}}{K_{f_{s}} \Delta_{m} \rho_{s}}}
$$

where $a$ is the stator mean radius, $K_{\mathrm{fs}}$ is the stator stacking factor, $\rho_{\mathrm{s}}$ is the stator stack mass density, and $\Delta_{\mathrm{m}}$ denotes the increasing mass because of the winding and teeth (details can be found in [13]).

Due to simplicity, the virtual displacements method is used to compute the elastic equilibrium. The stator of the studied PMSMs is defined as domain, where three forces such as 
surface $f_{\mathrm{s}}$, body forces $f_{\mathrm{b}}$, and concentrated forces $F^{\mathrm{i}}$. In a threedimensional plane, the mentioned forces which consist of all externally applied and reactions can be given as [21]:

$$
f^{s}=\left[\begin{array}{c}
f_{x}^{s} \\
f_{y}^{s} \\
f_{z}^{s}
\end{array}\right], \quad f^{B}=\left[\begin{array}{c}
f_{x}^{B} \\
f_{y}^{B} \\
f_{z}^{B}
\end{array}\right], \quad F^{i}=\left[\begin{array}{c}
F_{x}^{i} \\
F_{y}^{i} \\
F_{z}^{i}
\end{array}\right]
$$

By assuming that $\vec{u}$ vector is the displacement in the stator core, by defining $\vec{\varepsilon}$ as the strains of $\vec{u}$, and $\vec{\sigma}$ is the resulting stress from $\vec{\varepsilon}$. Hence, the virtual (small) displacement method solves the elasticity equilibrium based on the following equation [21]:

$$
\int_{V} \bar{\varepsilon}^{T} \sigma d V=\int_{V} \bar{u}^{T} f^{B} d V+\int_{S} \bar{u}^{s T} f^{S} d S+\sum_{i} \bar{u}^{i T} F^{i}
$$

\section{RESULTS AND DISCUSSION}

In this section, the most impactful vibro-acoustic parameters which are influenced by each machine are discussed to determine the best possible pole-pairs number for this specific PMSG. The input data of the studied machine is presented in Table II. The stator core contains 36 segmented teeth due to closed-slot modulation in order to reduce the amplitude of cogging torque significantly. The rotor consists of the different numbers of pole-pairs $(20,24,30,40$, and 42) in each machine. The rotor is modelled without the housing frame, which means the vibrating cylinder length is taken as total rotor yoke length. In the revised version, these pole-pairs numbers are chosen based on the double-layer non-overlapping FSCWs patterns. This type of winding is selected because of numerous benefits and recent improvements such as manufacturability and modularity, increased flux weakening performance, and short-end windings.

Each design case was simulated using variable speed MSSDM, via MANATEE. For the all studied PMSGs, the PMs are made of NdFeB-N48 with a remanent flux density of $1.2 \mathrm{~T}$, and the back irons are made of isotropic M400-50A non-orientated magnetic lamination steel with $7650 \mathrm{~kg} \cdot \mathrm{m}^{-3}$. The elastic characteristics of the lamination is $E_{\mathrm{x}}=215 \mathrm{MPa}, E_{\mathrm{y}}=215 \mathrm{MPa}$, and $E_{\mathrm{z}}=80 \mathrm{MPa}$, besides, shear modulus is $G_{\mathrm{xy}}=82.7 \mathrm{MPa}$, $G_{\mathrm{yz}}=2 \mathrm{MPa}$, and $G_{\mathrm{zx}}=2 \mathrm{Mpa}$. The Poisson ratio is defined as $V_{\mathrm{xy}}=0.3, V_{\mathrm{yz}}=0.03$, and $V_{\mathrm{zx}}=0.03$. Fig. 4 illustrates the nonlinear $B-H$ curve of the stator at different frequencies. As presented, a high risk of saturation in the teeth-tip can be seen when high magnetic field intensity between 700-1000 A/m exists. This phenomenon produces a magnetic bulk force, and thus, the importance of saturation role in overall magnetic force should be considered.

Table III presents the main slotting magnetic force characteristics, where $\mathrm{k}_{\mathrm{s}}$ and $\mathrm{k}_{\mathrm{r}}$ are integers involved in a permeance Fourier series. For $\pm \varepsilon$ which varies between -1 to 1 , the main slotting magnetic forces wavenumbers are calculated by $\mathrm{r}=\left(2 \times \mathrm{h}_{r}+1\right) \times \mathrm{p}+\varepsilon_{s} \times \mathrm{k}_{s} \times \mathrm{Q}_{s}+\varepsilon_{r} \times \mathrm{p}$, in which the

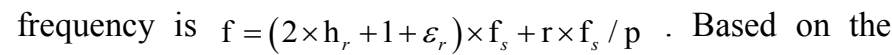
main $r$ and $f$, the resonance occurs (showed by 1 ) during flux weakening range at $420 \mathrm{rpm}$.

Fig. 5 presents the structure-based influence of each machine with a various number of pole-pairs on the A-weighted maximum sound power level $\left(W_{\mathrm{p}, \max }\right)$ magnitude in a variable speed analysis. The outer rotor structural modes are mostly characterized using the analogy with a cylindrical shell, whose structural modes can be labelled by $(m, n)$, where $m$ is the rank of the circumferential deflection and $n$ is the rank of the longitudinal deflection All studied PMSGs have produced four deflection modes in common such as outer rotor structure mode $(0,0),(4,0),(6,0)$ and $(8,0)$, in which each of these modes indicates a different structure deflection. Machine No. 1 (Fig. 5 (a)) has radiated a low $W_{\text {p, max }}$ by only $73.4 \mathrm{dBA}$ via model $(0,0)$, $(4,0)$, and $(8,0)$, if the machine operates under the ratings. At $435 \mathrm{rpm}$, the peak $W_{\text {p,max }}$ of $85 \mathrm{dBA}$ can be seen. Fig. 5(b) shows how increasing the pole-pairs number affects the machine structure deflection by only mode $(0,0)$. The maximum ASPL under the rated speed of $150 \mathrm{rpm}$ is $38.7 \mathrm{dBA}$, which is a considerable increase of $34.7 \mathrm{dBA}$ in comparison to the PMSG with 20 pole-pairs. A very high sensitivity of $W_{\mathrm{p}, \max }$ is apparent over the different pole-pairs number, even though this change is small. The $W_{\mathrm{p}, \max }$ radiated by machine No. 3 (Fig. 5(c)) is 53.4 $\mathrm{dBA}$ (under the rated speed of $150 \mathrm{rpm}$ ), from structure deflection modes $(0,0),(4,0)$, and $(8,0)$. Fig. 5(d) shows the maximum $W_{\mathrm{p}, \max }$ of $74.9 \mathrm{dBA}$ originated by deflection modes $(0,0),(4,0)$, and $(8,0)$ for machine No. 4. Fig. 5(e) presents the modal contribution of deflection modes $(0,0)$ and $(0,6)$, in which the maximum ASPL under rated speed is $72.3 \mathrm{dBA}$.

Fig. 6 illustrates the rotational operational deflection shapes (ODS)s of each machine's outer rotor (a sinusoidally fed slotted PMSG without PWM time harmonics and rotor MMF space harmonics), only resulting from its structure deflection modes $(m, n)$. To check the spatial order, the frequency and the propagation direction of pure slotting lines, the ODS analysis was done under a sinusoidal case $\left(f_{\mathrm{s}}=32.032 \mathrm{~Hz}\right)$ on a 12-point mesh near the rotor frame median circumference. The accelerometer precision and the software features enable to visualize rotor structure movements at each individual frequency, even when it is subjected to an exciting force far

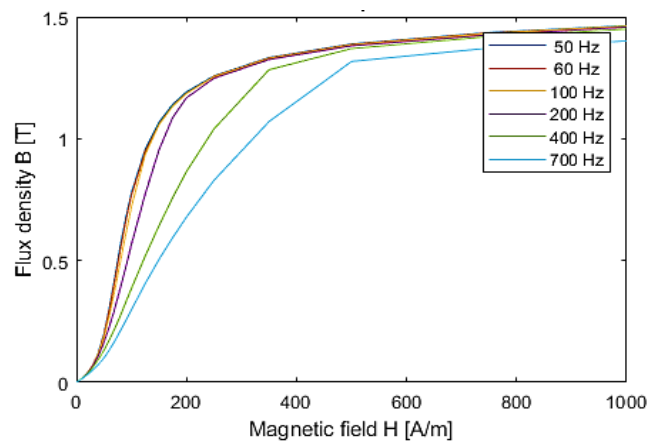

Fig. 4 Nonlinear $B-H$ curve of the stator with steel lamination of M400-50A

TABLE II. INPUT DATA OF THE STUDIED PMSG

\begin{tabular}{l|lll}
\hline Parameters & Description & Values & Units \\
\hline$l_{s}$ & Stack length & 100 & $\mathrm{~mm}$ \\
$R_{r i} / R_{r o}$ & Rotor radiuses & $217 / 230$ & $\mathrm{~mm}$ \\
$R_{s i} / R_{s o}$ & Stator radiuses & $115 / 209.5$ & $\mathrm{~mm}$ \\
$S_{w}$ & Slot width & 15 & $\mathrm{~mm}$ \\
$\delta_{g}$ & Air-gap length & 0.6 & $\mathrm{~mm}$ \\
$S_{d}$ & Slot depth & 50 & $\mathrm{~mm}$ \\
$Q_{s} / 2 P$ & Stator slot/ poles & $36 / 20$, etc & \\
$\alpha_{p}$ & Pole pitch & 0.55 & \\
$S P$ & Slot pitch & 0.9 & \\
$\mu_{0}$ & Vacuum permeability & $4 \pi \times 10^{-7}$ & $\mathrm{H} / \mathrm{m}$ \\
$\mu_{\mathrm{s}}$ & Iron relative permeability & 2500 & \\
$\mu_{\mathrm{PM}}$ & Magnet relative permeability & 1.05 & \\
$\mu_{\mathrm{air}}$ & Air relative permeability & 1 & \\
$B_{\mathrm{rm}}$ & Magnet residual flux density & 1.2 & $\mathrm{~T}$ \\
\hline
\end{tabular}



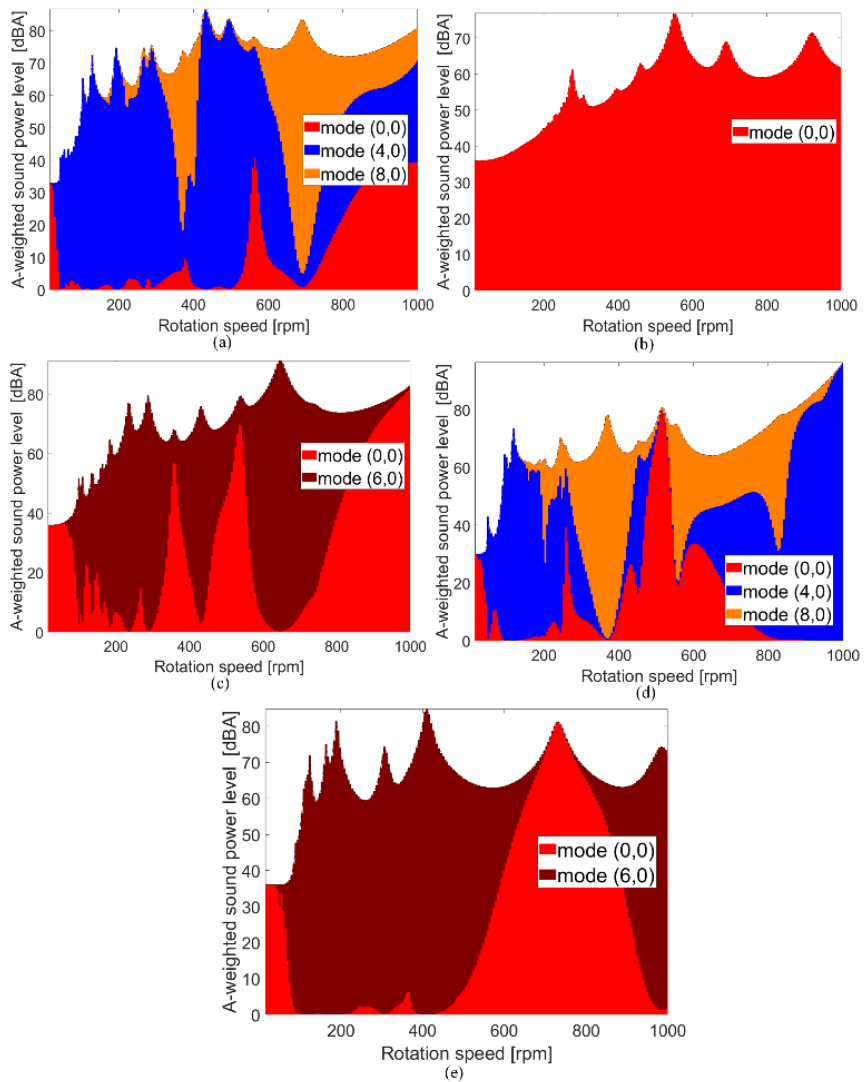

Fig. 5. Modal contribution of the machine structure on the sound power level radiation, (a) Machine No.1, (b) Machine No. 2, (c) Machine No. 3, (d) Machine No. 4, and (e) Machine No. 5.

from any resonance. Fig. 6(a) shows the ODS with counterclockwise rotation (CCR) of machine No. 1 which is only caused by modes $(0,0),(4,0)$, and $(8,0)$, where the rotor and stator main slotting frequencies are $Z_{r} * f_{R}=100 \mathrm{~Hz}$, and $Z_{s} * f_{R}$ $=90 \mathrm{~Hz}$. Fig (without resonance effects). 6(b) presents the ODS with CCR originating from mode $(0,0)$ on machine No. 2 . The rotor and stator main slotting frequencies are $50 \mathrm{~Hz}$, and $90 \mathrm{~Hz}$. Fig. 6(c) represents the ODS with clockwise (CR) of machine No. 3 which is from mode $(0,0)$ and $(6,0)$, where the rotor and stator main slotting frequencies are $75 \mathrm{~Hz}$, and $90 \mathrm{~Hz}$. Fig. 6(d) shows the ODS with CCR of machine No. 4 originated by modes $(0,0),(4,0)$, and $(8,0)$, where the rotor and stator main slotting frequencies are $100 \mathrm{~Hz}$, and $90 \mathrm{~Hz}$. Fig. 6(e) depicts the ODS of machine No. 5 with CR by modes $(0,0)$ and $(6,0)$. The rotor and stator main slotting frequencies are $105 \mathrm{~Hz}$ and $90 \mathrm{~Hz}$. It can be seen that each machine has its own specific ODS, regardless of the type of structure modes involved. All ODSs are simulated under different slotting force waves and their propagation direction (as summarized in Table III).

Fig. 7 presents the overall vibrations over variable speed of each PMSG model, in which the dashed line shows the rated speed of $150 \mathrm{rpm}$. As presented in the graph, machine No. 1 produces maximum vibration of $119.6 \mathrm{~dB}$ at $135 \mathrm{rpm}$. Machine No. 2 radiates the lowest vibration by $81.5 \mathrm{~dB}(\max )$ at $150 \mathrm{rpm}$. Machine No. 3 with 30 poles radiates a maximum vibration of $100 \mathrm{~dB}$ at $150 \mathrm{rpm}$. The highest vibration is produced by machine No. 4 (40 poles) at $122 \mathrm{~dB}$. A lower level of vibration is reported $(108.6 \mathrm{~dB}$ at $130 \mathrm{rpm})$ by machine No. 5 , where 42 poles are used. Above the rated speed, up to $1000 \mathrm{rpm}$, the vibration level gradually increases, where machine No. 4 obtains $144 \mathrm{~dB}$ at $1000 \mathrm{rpm}$ as the highest, while the lowest vibration of $115 \mathrm{~dB}$ at $573 \mathrm{rpm}$ is radiated by machine No. 2 .

Fig. 8 depicts A-weighted, maximum magnetic sound power level $W_{\mathrm{p}}$ sonograms of different numbers of pole-pairs under a variable speed range, evaluated in the post-processing stage. The electromagnetically-excited radial vibration illustration identifies whether, at variable speeds of the PMSG, the vibration resonances caused by magnetic forces occur during the operation or not. The designed PMSGs allow for operation under $150 \mathrm{rpm}, 50 \mathrm{~Hz}$, while it is a low-speed wind generator. In Fig. 8(a), machine No. 1 with 20 pole-pairs has been simulated with $60.6 \mathrm{dBA}$ at $150 \mathrm{rpm}$, and $86.81 \mathrm{dBA}$ (up to $1000 \mathrm{rpm})$. The first major resonances actually occur around $48 \mathrm{~Hz}$ at $288 \mathrm{rpm}$ (marked as 1), $95 \mathrm{~Hz} 563 \mathrm{rpm}$ (marked as 2), $101 \mathrm{~Hz}$ at $605 \mathrm{~Hz}$ (marked as 3), and $115 \mathrm{~Hz}$ at $691 \mathrm{rpm}$, which are also shown by the red dashed lines. The while ellipsoids indicate the critical noisy areas (above $75 \mathrm{dBA}$ ). The main slotting magnetic forces wavenumbers of $r=0,4,-4,8$, and -8 are produced in this machine. The noisy areas occur mostly under $1000 \mathrm{rpm}$. Fig. 8(b) presents the $W_{\mathrm{p}}$ (or magnet noise)

TABLE III. MAIN SLOTTING MAGNETIC ForCE FEATURES

\begin{tabular}{c|ccccccc}
\hline No. & $k_{s}$ & $k_{r}$ & $\varepsilon_{\mathrm{s}}$ & $\varepsilon_{\mathrm{r}}$ & $r$ & Frequency [Hz] & Resonance \\
\hline 1 & 1 & 1.8 & -1 & \pm 1 & +4 & 57.6 & 0 \\
2 & 2 & 3.6 & -1 & \pm 1 & +8 & 115.3 & 0 \\
3 & 8 & 14.4 & -1 & \pm 1 & -8 & 461.3 & 1 \\
4 & 9 & 16.2 & -1 & \pm 1 & -4 & 518.9 & 1 \\
5 & 10 & 18 & -1 & \pm 1 & 0 & 576.5 & 1 \\
\hline
\end{tabular}
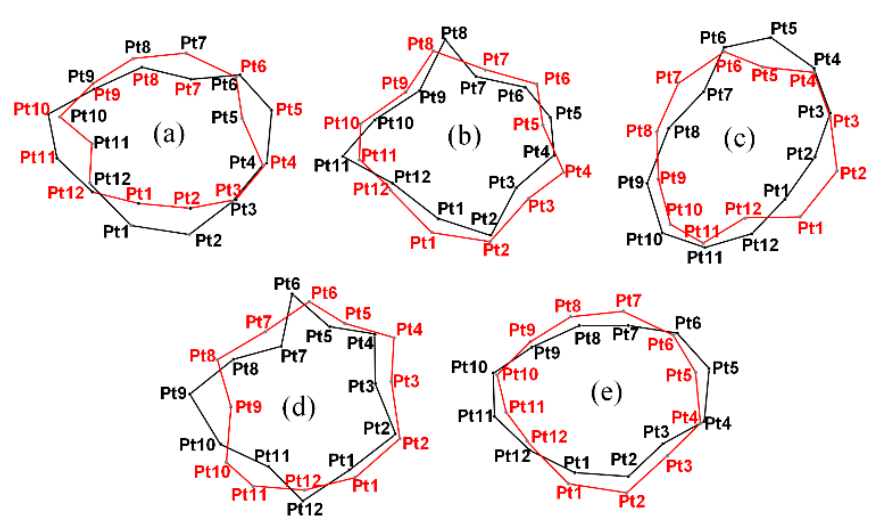

Fig. 6. Outer rotor ODSs visualization of the outer rotor with considering slotting force waves, and their propagation direction (counter-clockwise rotation (CCR), and clockwise rotation (CR)), (a) Machine No.1, (b) Machine No. 2, (c) Machine No. 3, (d) Machine No. 4, and (e) Machine No. 5.

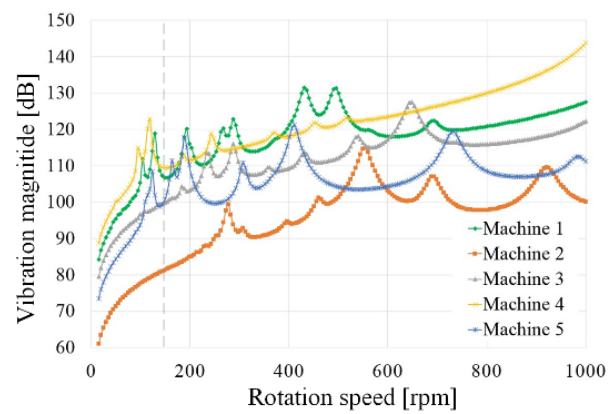

Fig. 7. Overall vibration under variable speed analysis, (a) Machine No.1, (b) Machine No. 2, (c) Machine No. 3, (d) Machine No. 4, and (e) Machine No. 5. 
sonogram of machine No. 2 (36slots/24poles) as $40.62 \mathrm{dBA}$ at $150 \mathrm{rpm}$ and $76.89 \mathrm{dBA}$ (up to 1000rpm), in which a considerably lower magnetic noise is radiated in comparison to machine No. 1. In addition, the main slotting magnetic forces wavenumber of $r=0$ is generated in this machine. The main resonances are addressed by the red dashed lines at $111 \mathrm{~Hz}$ and $553 \mathrm{rpm}$ (marked as 1), $138 \mathrm{~Hz}$ and $691 \mathrm{rpm}$ (marked as 2), and $184 \mathrm{~Hz}$ and $921 \mathrm{rpm}$ (marked as 3). As presented, the number of noisy spots are much lower than machine No.1. The noisiest areas are reported over $1200 \mathrm{rpm}$. Fig. 8(c) shows the variable speed magnetic noise sonogram of machine No. 3 (36slots/ 30 poles) by with $58.5 \mathrm{dBA}$ at $150 \mathrm{rpm}$, and $70.55 \mathrm{dBA}$ (up to $1000 \mathrm{rpm}$ ), in which the main resonances have occurred at 107 $\mathrm{Hz}$ and $295 \mathrm{rpm}$ (marked as 1), $115 \mathrm{~Hz}$ and $446 \mathrm{rpm}$ (marked as 2), $121 \mathrm{~Hz}$ and $535 \mathrm{rpm}$ (marked as 3), and $143 \mathrm{~Hz}$ and 649 rpm (marked as 4). The main slotting magnetic forces wavenumbers of $r=0,6$, and -6 are involved in this machine. Fig. 8(d) indicates the $W_{\mathrm{p}}$ sonogram of machine No. 4 (36slots/ 40poles) as $62.06 \mathrm{dBA}$ at $150 \mathrm{rpm}$ and $96.39 \mathrm{dBA}$ (from 15 to $1000 \mathrm{rpm}$ ). The main slotting magnetic forces wavenumbers of $r=0,4$, and 8 are involved in this machine. There are two main resonances marked as 1 and 2, which have occurred at $172 \mathrm{~Hz}$ and $516 \mathrm{rpm}$, as well as $185 \mathrm{~Hz}$ and $554 \mathrm{rpm}$. Fig. 8(e) represents the magnetic noise of machine No. 5 (36 slots/ 42poles) as $62.24 \mathrm{dBA}$ at $150 \mathrm{rpm}$ and $84.85 \mathrm{dBA}$ from $15-1000$ $\mathrm{rpm}$. The main slotting magnetic forces wavenumbers of $r=0$, 6 , and -6 are involved in this machine.

The main resonances are addressed by the red dashed lines as a function of speed and frequency, which are $108 \mathrm{~Hz}$ and 308 rpm (marked as 1), $144 \mathrm{~Hz}$ and $410 \mathrm{rpm}$ (marked as 2), and 256 $\mathrm{Hz}$ and $731 \mathrm{rpm}$ (marked as 3). Also, major magnetic noises are radiated over $1000 \mathrm{rpm}$. The frequency response function of Maxwell stress wavenumbers (e.g. $r=2$ meets the natural frequencies of the elliptical model $(2,0))$ of the PMSG.

Table IV compares the outer rotor natural frequencies based on the modal damping coefficients approach of all the PMSG models (M.1 to M.5) with different pole numbers. The response of the outer rotor under magnetic forces has been quantified using the rotor (including magnets) excited through radial and tangential elementary forces. The rules of the switching frequency are based on [13, Eq. 10 and 11], these rules can either be applied prior to the PWM strategy setup. To reduce magnetic noise, the PMSG natural frequencies must be properly designed in terms of the switching strategy.

Table $\mathrm{V}$ presents a comparison of the three main vibroacoustic and electromagnetic outputs of all the studied PMSG (M.1 to M.5). Three first rows present the harmonic errors $f_{1}, f_{2}$, and $f_{3}$ of acoustic amplitude using MS-SDM, where the magnetic force in the airgap is assumed as the reference. The errors are less than $3 \%$ which is ignorable. Among all the models, M. 1 has produced both the lowest electromagnetic (output power, torque, and efficiency) and above average vibroacoustic outputs, $106.7 \mathrm{~dB}$ vibration and $60.5 \mathrm{dBA}$ magnetic noise. M. 2 has radiated the lowest vibrations and magnetic noise $(81.3 \mathrm{~dB}$ and $40.6 \mathrm{dBA})$, however the output power, torque and efficiency $(91.6 \%)$ have been reduced. M. 3 has performed more reliable in both electromagnetic and vibroacoustic outputs, where the magnetic noise is $58.5 \mathrm{dBA}$ and

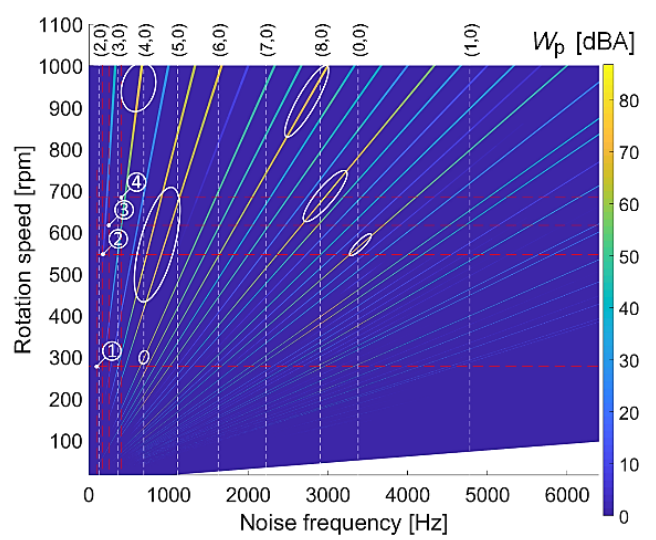

(a)

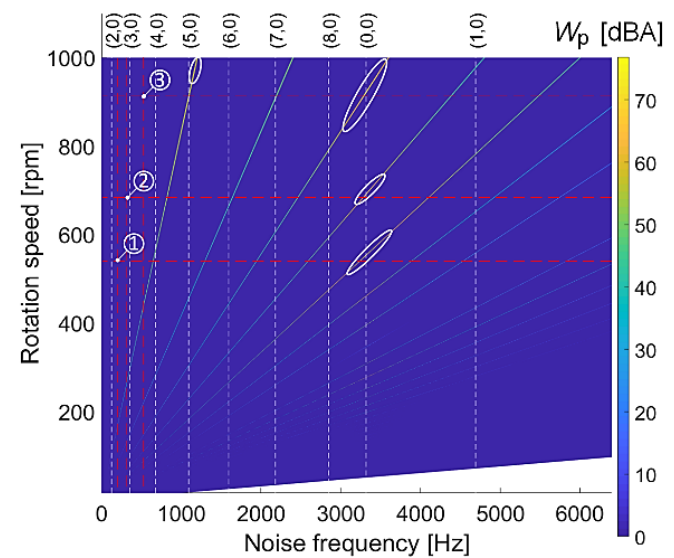

(b)

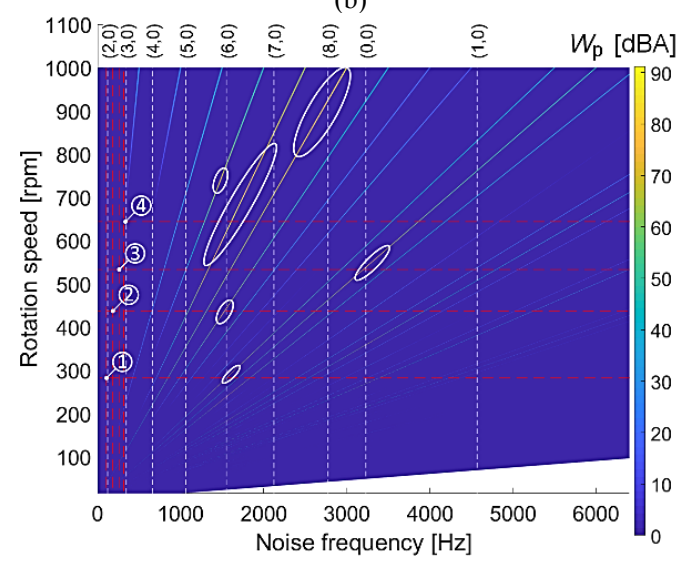

(c)

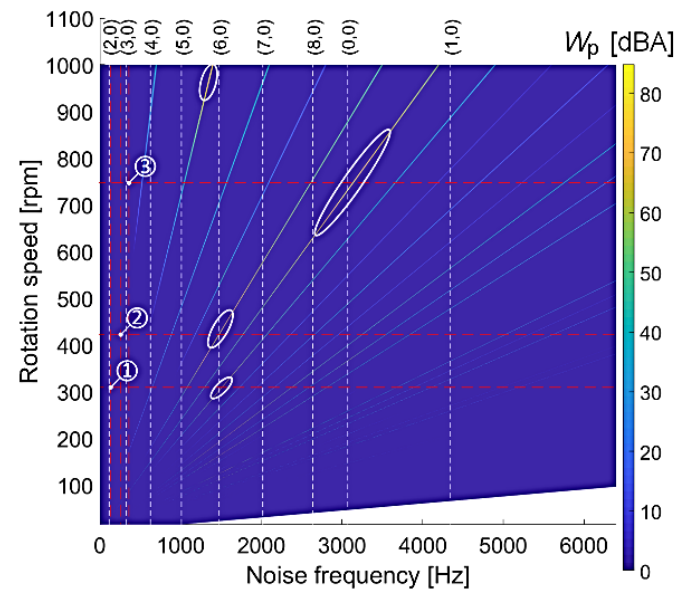

(d) 


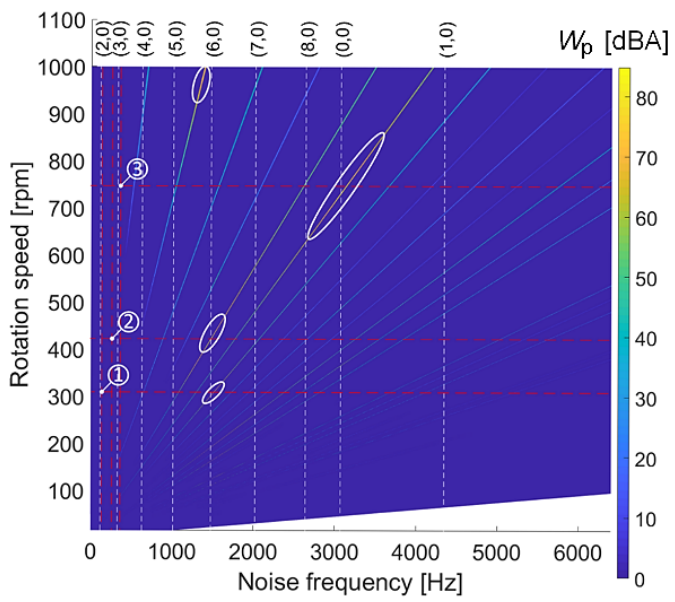

(e)

Fig. 8. A-weighted maximum sound power level ( $\left.W_{\mathrm{p}, \max }\right)$ sonograms of PMSGs under variable speed analysis and stator natural frequencies consideration, (a) Machine No.1, (b) Machine No. 2, (c) Machine No. 3, (d) Machine No. 4, and (e) Machine No. 5.

efficiency of the PMSG, 94.7\%. Therefore, the 30 pole-pairs number is a better choice than 20 and 24 pole-pairs. M. 4 has produced rather higher vibration and magnetic noise (109.44), but the electromagnetic outputs have a significantly very high efficiency of $97.1 \%$. M. 5 shows still a very high electromagnetic performance, in which the efficiency of $96.408 \%$ is obtained. Also, the vibrations and magnetic noise radiations are lower than $M .1$, and $M$. 4. In general, as the PMSG is not producing a critical level of vibrations and magnetic noise, M. 4 and M. 5 perhaps can be suitable because of very high electromagnetic performance. The choice between these two machines ( 40 or 42 poles) is a tradeoff issue for the designer.

TABLE IV. ROTOR NATURAL FREQUENCIES OF THE PMSGS

\begin{tabular}{c|cccccc}
\hline$m$ & Unit & $M .1$ & $M .2$ & $M .3$ & $M .4$ & $M .5$ \\
\hline 0 & $\mathrm{~Hz}$ & 3379 & 3317 & 3188 & 3096 & 3072 \\
1 & $\mathrm{~Hz}$ & 4779 & 4690 & 4505 & 4379 & 4344 \\
2 & $\mathrm{~Hz}$ & 128 & 125 & 120 & 117 & 116 \\
3 & $\mathrm{~Hz}$ & 368 & 354 & 342 & 331 & 328 \\
4 & $\mathrm{~Hz}$ & 960 & 678 & 653 & 633 & 628 \\
5 & $\mathrm{~Hz}$ & 1112 & 1091 & 1018 & 1019 & 1011 \\
6 & $\mathrm{~Hz}$ & 1623 & 1593 & 1516 & 1488 & 1476 \\
7 & $\mathrm{~Hz}$ & 2221 & 2180 & 2109 & 2036 & 2020 \\
8 & $\mathrm{~Hz}$ & 2902 & 2849 & 2751 & 2661 & 2640 \\
\hline
\end{tabular}

TABLE V. ELECTROMAGNETIC-VIBRO-ACOUSTIC RESULTS AND COMPARISON AT 150RPM

\begin{tabular}{c|ccccc}
\hline Parameters & $M .1$ & $M .2$ & $M .3$ & $M .4$ & $M .5$ \\
\hline Harm. error $f_{1}[\mathrm{~dB}]$ & -0.1 & -0.08 & +0.25 & +0.04 & +0.05 \\
Harm. error $f_{2}[\mathrm{~dB}]$ & +0.8 & +1.1 & -3.1 & +2.7 & +1.9 \\
Harm. error $f_{3}[\mathrm{~dB}]$ & +1.5 & +1.3 & +3.9 & +2.2 & +2.5 \\
Vibration $[\mathrm{dB}]$ & 106.72 & 81.399 & 100 & 109.44 & 101.08 \\
$L_{\mathrm{p}, \max }[\mathrm{dBA}]$ & 60.5 & 40.626 & 58.5 & 62.065 & 62.249 \\
$W_{\mathrm{p}}[\mathrm{dBA}]$ & 50.72 & 30.846 & 49.3 & 52.285 & 52.469 \\
$P_{\mathrm{o}}[\mathrm{kW}]$ & 1.391 & 1.591 & 4.172 & 5.5159 & 4.9991 \\
$\eta[\%]$ & 90.6 & 91.6 & 94.71 & 97.163 & 96.408 \\
$T[\mathrm{~N} . \mathrm{m}]$ & 99 & 102 & 290.29 & 378.93 & 346.872 \\
\hline
\end{tabular}

\section{3-D FEA AND EXPERIMENTAL VERIFICATIONS}

The manufactured prototypes (M. 5) with the optimum pole numbers of 42 is tested for the case of small wind power generation $(5 \mathrm{~kW})$, under EU directive 2010/30/EU sound power level regulation. The sound meter used to gather noise data is the model SC-420 from CESVA. This device allows to measure sounds and noises in different frequency filters. For this project the option used allows for the reduction of ambient sounds and those noises that are external to the noise source being measured.

Fig. 9 indicates both the normal and centrifugal forces $\left(F_{\mathrm{r}}\right.$ and $F_{\mathrm{t}}$ ), in which both values are originated from the magnetic field analysis (radial and tangential components of magnetic field $\left.B_{\mathrm{n}}, B_{\mathrm{t}}\right)$. The blue curve shows $F_{\mathrm{r}}$ using MS-SDM method, red curve (as reference) $F_{\mathrm{r}}$ using 3-D FEA. Note that both $F_{\mathrm{r}}$ curves are aligned by the left-side vertical axis. The green (MSSDM) and purple (3-D FEA) curves indicate $F_{\mathrm{t}}$ which is aligned by the right-side vertical axis. Fig. 9(b) depicts the FFT amplitude of those curves, where $f_{\mathrm{n}}$ shows the largest harmonic content after fundamental.

Fig. 10 illustrates the test bench setup at the Machine Lab of Universitat Politècnica de Catalunya. The two main measurement kits are used, CESVA SC-420 (magnetic noise), and Rion AV-12 for the vibrations. To gather noise data of the PMSG under rated speed, the PMSG was placed, based on ISO 6396. The microphone has been placed in the radial direction of the rotor $60 \mathrm{~cm}$ away (as the same as simulation setup) from the PMSG to reduce the effect from the airflow, which is induced by the rotating rotor.

Fig. 11 validates the magnetic noise of machine No. 5 as the function of rotation speed with peak value $73.8 \mathrm{dBA}$ (blue curve) for speed range between 15-150 rpm, which has been experimentally verified as $82.1 \mathrm{dBA}$ for the M. 5 with 42 poles. During the test, the rotor of the studied PMSG was coupled to an induction motor for deriving purpose, the induction motor itself produced between 5 to $15 \mathrm{dBA}$ (these values are measured

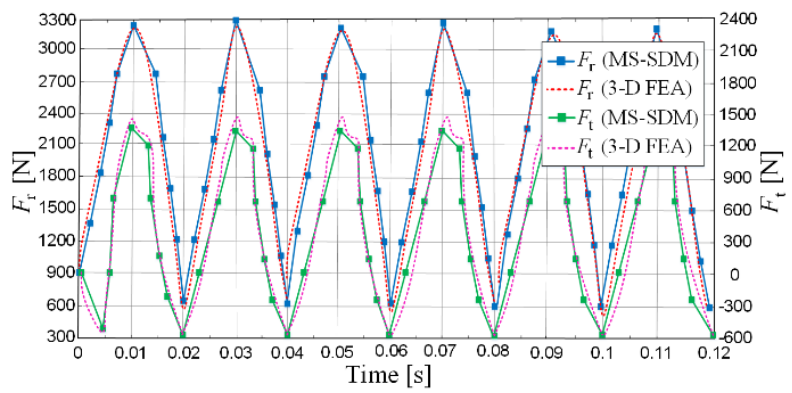

(a)

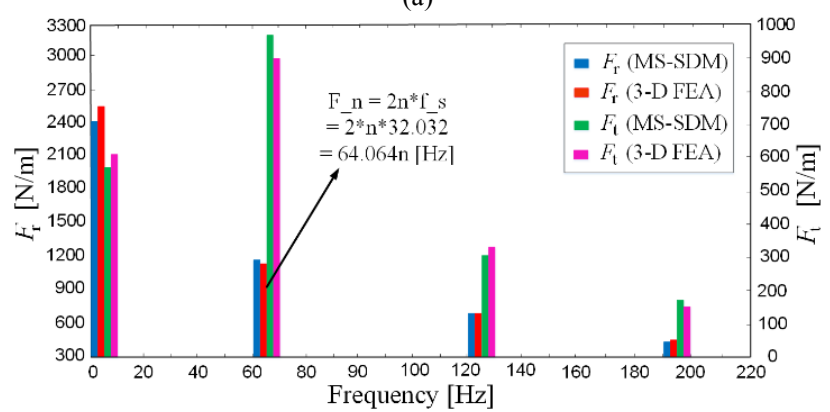

(b)

Fig. 9 Airgap magnetic forces distribution of the PMSM with 36 slots and 40 poles using proposed MS-SDM and 3-D FEA, (a) $F_{\mathrm{r}}$ and $F_{\mathrm{t}}$, and (b) FFT magnitude of each component. 
when the induction motor was operating without coupling with other machines) between $0-1000 \mathrm{rpm}$. As presented, the grey curve shows the experimental maximum noise measurement when the machine driver (induction motor) noise is cancelled. The difference between MS-SDM method and experimental measurement (grey curve) is less than $5 \mathrm{dBA}$ (the only source of this emitted noise is environmental sounds) under rotation speed of $200 \mathrm{rpm}$.

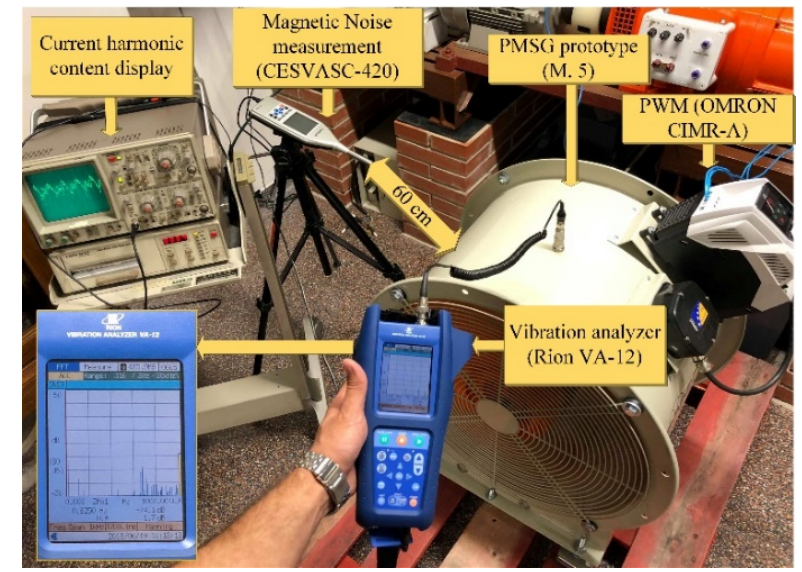

Fig. 10. The experimental bench setup.

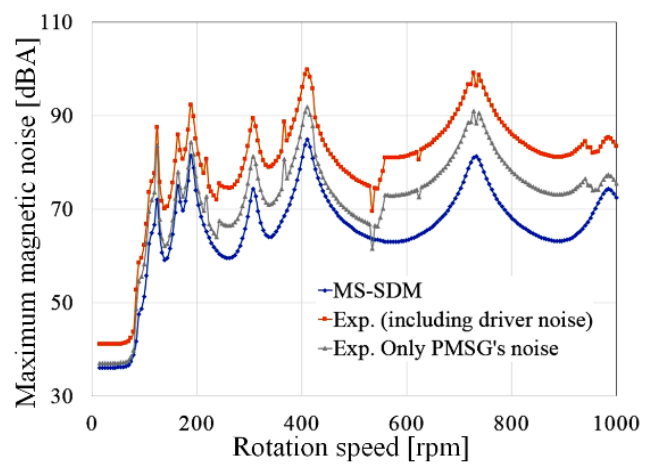

Fig. 11. Variable speed A-weighted sound power level validation.

\section{CONCLUSION}

This work has studied a radial-flux surface mounted permanent magnet synchronous generator (PMSG) with outer rotor topology, an even integer slot number (in this case 36), which is wound with a double-layer non-overlapping FSCW. This slot number has produced even force wavenumbers $(0,2$, 4, 6, and 8) of Maxwell force harmonics under a variable-speed computation of electromagnetically-excited noise to determine the predicted vibro-acoustic radiations. To find the optimal pole number, five PMSGs with double-layer non-overlapping FSCW and different pole numbers (such as 20, 24, 30, 40, and 42) were modeled. To compute the vibro-acoustic performance of each studied PMSG, all the simulations were completed using MS-SDM for higher accuracy in comparison to the conventional SDM which neglects skewed parts. After a careful study on the MMF harmonic analysis, variable speed vibration spectrums and the magnetic noise while considering the natural frequencies; we have found the PMSG with 42 poles (M. 5) with a better performance in both electromagnetic and magnetic noise, where slot per pole per phase is the smallest $(q=0.285)$, and higher number of poles is used which refers to higher GCD $\left(Q_{\mathrm{s}}, 2 p\right)$. Finally, the semi-analytical results from MS-SDM on M. 5 have been validated using both 3-D FEA and experimental measurements. In contrast, the use of MS-SDM enables a considerable decrease in time computation of each model (average value of 13.349s) by approximately eight times faster than 3 -D FEA by average value of $112.39 \mathrm{~s}$. The error between both models is less than $\pm 2.5 \%$.

\section{REFERENCES}

[1] G. Kron, "Induction motor slot combinations: Rules to predetermine crawling vibration, noise and hooks in the speed-torque curve," AIEE Trans., vol. 50, 1931.

[2] P. Timar, Noise and Vibration of Electrical Machines. New York: Elsevier, 1989.

[3] J.F. Gieras, C. Wang and J.C. Lai, Noise of polyphase electric motors, CRC Press, 2005

[4] P. Juha, Tapani Jokinen, Valéria Hrabovcova, Design of Rotating Electrical Machines (2nd Ed.), Wiley, 2013.

[5] C-Min Lee, H-Soo Seol, J-y. Lee, S-H. Lee, and D-Woo Kang, "Optimization of Vibration and Noise Characteristics of Skewed Permanent Brushless Direct Current Motor," IEEE Trans. Magn. Vol. 53, No. 11, pp. 1-5, 2017.

[6] S. Guy Min, and B. Sarlioglu, "Modeling and Investigation on Electromagnetic Noise in PM Motors With Single- and Double-Layer Concentrated Winding for EV and HEV Application," IEEE Trans. Transportation Electrification, Vol. 4, No. 1, pp. 292-302, Mar. 2018.

[7] W. Deng, S. Zuo, F. Lin, S. Wu, "Influence of pole and slot combinations on vibration and noise in external rotor axial flux in-wheel motors," IET Electr. Power Appl., Vol. 11, No. 4, pp. 586-594, 2017.

[8] G-Yu Zhou and J-Xin Shen, "Rotor Notching for Electromagnetic Noise Reduction of Induction Motors," IEEE Trans. Ind. Appl. Vol. 53, No. 4, pp. 3361-3370, Nov. 2017.

[9] T. Lubin, S. Mezani, and A. Rezzoug, "2-D exact analytical model for surface-mounted permanent-magnet motors with semi-closed slots," IEEE Trans. Magn., vol. 47, no. part 2, pp. 479-492, 2011.

[10] Z. Q. Zhu, Z. P. Xia, L. J. Wu, and G. W. Jewell, "Analytical modeling and finite-element computation of radial vibration force in fractional-slot permanent-magnet brushless machines," IEEE Trans. Ind. Appl., vol. 46, no. 5, pp. 1908-1918, 2010.

[11] J. Le Bensnerais, V. Lanfranchi, M. Hecquet, and P. Brochet, "Optimal Slot Numbers for Magnetic Noise Reduction in Variable-Speed Induction Motors," IEEE Trans. Magn., vol. 45, no. 8, pp. 3131-3136, Aug. 2009.

[12] J. Le Bensnerais, V. Lanfranchi, M. Hecquet, and P. Brochet, "Characterization and Reduction of Audible Magnetic Noise Due to PWM Supply in Induction Machines," IEEE Trans. Ind. Electron., vol. 57, no. 4, pp. 1288-1295, Apr. 2010.

[13] J. Le Bensnerais, V. Lanfranchi, M. Hecquet, G. Friedrich, and P. Brochet, "Characterisation of radial vibration force and vibration behaviour of a pulse-width modulation-fed fractional-slot induction machine," IET Electr. Power App., vol. 3, no. 3, pp. 197-208, May. 2009.

[14] M. Liwschitz-Garik and C. C. Whipple, Electric Machinery, vol. II, A-C Machines. New York: D. Van Nostrand Company Inc., 1960.

[15] N. Bianchi and M. D. Pré, "Use of the star of slots in designing fractionalslot single-layer synchronous motors," IEE Proc. - Electr. Power Appl., vol. 153 , no. 3, pp. 459-466, May 2006, (online no. 20050284).

[16] H. A. Toliyat, and G. B. Kliman, "Handbook of Electric Motors," CRC press/ Taylor \& Francis Group, ISBN: 0-8247-4105-6, 2004.

[17] J. Li, Da-W. Choi, D-H. Son, and Y-Hyun Cho, "Effects of MMF Harmonics on Rotor Eddy-Current Losses for Inner-Rotor Fractional Slot Axial Flux Permanent Magnet Synchronous Machines," IEEE Trans. Magnetics, Vol. 48, No. 2, Feb. 2012.

[18] E. Devillers, J. Le Besnerais, T. Lubin, M. Hecquet, and J. P. Lecointe, "A review of subdomain modeling techniques in electrical machines: performances and applications," in ICEM International Conference on Electrical Machines, 2016.

[19] C. Xia, Z. Zhang, and Q. Geng, "Analytical Modeling and Analysis of Surface Mounted Permanent Magnet Machines with Skewed Slots," IEEE Trans. Magn., vol. 51, no. 5, pp. 1-8, May. 2015.

[20] Belahcen, "Magnetoelasticity, magnetic forces and magnetostriction in electrical machines," $\mathrm{PhD}$ thesis, Helsinki University of Technology, Finland, 2004 